\title{
Desafíos del nuevo constitucionalismo en América Latina ${ }^{* *}$
}

\section{Resumen}

Partiendo del supuesto de que las constituciones surgen en contextos convulsionados política y socialmente, como fruto del malestar existente, o, por el contrario, como faros de esperanza de los pueblos y naciones que las promulgan, el autor analiza el origen del constitucionalismo en América Latina y precisa los desafíos que éste actualmente tiene, ilustrando con realidades específicas de algunos países de la región.

\section{Palabras clave}

Desafíos, constitucionalismo, América Latina.

* Doctor en

Derecho por la

Universidad

Complutense

de Madrid,

Investigador

del Instituto de

Investigaciones

Juridicas de la

Universidad

Nacional

Autónoma de

México.

\section{Introducción.}

Cada proceso constituyente responde a circunstancias muy diversas. Aunque es cierto que los problemas que se pretenden resolver o enfrentar a través de la expedición de nuevas constituciones son parecidos, los impulsos que las hacen surgir son particulares de cada país e incluso de cada momento histórico. Importa mucho el sentido que le quiere dar cada generación constituyente a su obra, el cual siempre estará marcado -en un grado mayor o menor- por las circunstancias que rodean al proceso de discusión y redacción de las nuevas constituciones.

Si revisamos la historia del Estado constitucional podremos corroborar que las circunstancias a través de las que han podido ir surgiendo las constituciones de nuestro tiempo (es decir, las constituciones en sentido moderno, las que se comienzan a expedir a finales del siglo XVIII), han sido muy variables. No hay una receta única e infalible para saber cuándo una Constitución podrá aspirar con cierto fundamento a tener una 
larga vida o cuando caerá ante el primer soplo de oposición y será reemplazada por otra igualmente efímera.

Tampoco se puede saber con certeza qué contenido saldrá de una convención constituyente: si la Constitución será progresista o conservadora, si supondrá un avance fenomenal para el país de que se trate o si será una vuelta a la caverna. Nada está escrito de una vez y para siempre en la historia (pasada, presente y futura) del Estado constitucional ${ }^{1}$.

La circunstancia que parece acompañar a un número importante de procesos constituyentes en la historia es una suerte de crisis en los fundamentos de las respectivas sociedades, o al menos en la forma de ejercer y repartir el poder.

Las Constituciones surgen, pues, en contextos convulsionados política y socialmente, como fruto del malestar existente, pero también como faros de esperanza de los pueblos y naciones que las promulgan; esperanza en el derecho, en la justicia, en la posibilidad de construir sociedades nuevas, basadas en el respeto a los derechos de todos y en una convivencia futura de carácter pacífico. ¿o es esa, finalmente, la meta de todo sistema jurídico? Pues nada mejor que comenzar a construirla precisamente desde el fundamento mismo de los ordenamientos jurídicos modernos: las Constituciones.

\section{Historias, constituciones y utopías.}

Cuando surge la primera Constitución en sentido moderno, la que fue creada en Filadelfia en 1787 (todavía vigente, más de 220 años después), lo que se buscaba era fundar una nación independiente. De hecho, el documento que sirve de plataforma ideológica de la Constitución norteamericana es precisamente la famosa Declaración de Independencia, promulgada en 1776.

Uno de los aspectos más importantes de su contenido y sobre todo de su sentido es quizá que la Declaración resume una filosofía política que será la que oriente hacia el futuro el entendimiento de los derechos fundamentales en Estados Unidos, conformando de esa manera el influyente "modelo americano de derechos", que hoy en día es quizá el que mayor interés pueda tener desde la óptica del derecho constitucional comparado.

El texto de la Declaración fue elaborado por una comisión de la que formaron parte personajes tan importantes como John Adams, Benjamín Franklin y Thomas Jefferson. La redacción inicial del documento correspondió justamente a Jefferson. Tuvo como antecedente un documento previo, redactado por Henry Lee y aprobado el 7 de junio

\footnotetext{
Este texto tiene su origen en la participación del autor en el Encuentro Internacional: "El nuevo constitucionalismo: desafios y retos para el siglo XXI", organizado por la Corte Constitucional de Ecuador, Quito, 22 de octubre de 2009 y en el I Encontro Iberoamericano de Direito Constitucional, organizado por el Instituto Brasileiro de Direito Constitucional, Sao Paulo, 29 de octubre de 2009.

1 Ver, al respecto, las reflexiones de Zagrebelsky, Gustavo, Historia y Constitución, Madrid, Trotta, 2005.
} 
de 1776, en el que las colonias ya manifestaban el deseo de separarse de Inglaterra. El documento de junio iba dirigido específicamente al monarca inglés y a toda Gran Bretaña. La Declaración del 4 de julio tenía una ambición mayor: se dirigía a toda la humanidad, avisando del surgimiento de una nueva nación soberana ${ }^{2}$.

El texto redactado por Jefferson fue objeto de modificaciones de estilo por parte de Adams y Franklin. Incluía originalmente una cláusula que condenaba la esclavitud y hacía recaer la responsabilidad por el inhumano tráfico de personas de color en el monarca inglés, pero tuvo que ser removida por las protestas de Carolina del Sur y Georgia, que estaban de acuerdo en mantener el esclavismo.

En la redacción de la Constitución estadounidense influyen muchos pensadores y varias corrientes ideológicas, las cuales defendían conceptos distintos e incluso opuestos sobre lo que tenía que ser una Constitución. Ahí estaban -nuevamente- las ideas de Thomas Jefferson sobre el gobierno de las generaciones vivas y la necesidad de proceder a cambiar la Constitución cada vez que una nueva generación subiera al poder; pero también se hizo presente la influencia de James Madison, que quería una Constitución para la eternidad, un documento que estuviera prácticamente escrito en piedra ${ }^{3}$.

Es precisamente en una carta que Jefferson escribe a Madison donde mejor se plasma su idea de que "la tierra pertenece a los vivos". Escribiendo desde París, donde era embajador de los Estados Unidos, Jefferson le dice a Madison en una misiva del 6 de septiembre de 1789 que "los vivos tienen la tierra en usufructo; y los muertos no tienen poder ni derechos sobre ella. La porción que ocupa un individuo deja de ser suya cuando él mismo ya no es, y revierte a la sociedad... ninguna sociedad puede hacer una constitución perpetua, ni tan siquiera una ley perpetua. La tierra pertenece siempre a la generación viviente: pueden, por tanto, administrarla, y administrar sus frutos, como les plazca, durante su usufructo... toda constitución, y toda ley, caducan naturalmente pasados treinta y cuatro años" ${ }^{4}$.

En respuesta a Jefferson, también por medio de una carta, Madison expresa sus dudas sobre la conveniencia de reformar con tanta frecuencia un texto constitucional o de darlo por caducado por el simple relevo generacional. Se pregunta Madison en una carta del 4 de febrero de 1790: “¿Un Gobierno reformado con tanta frecuencia no se

\footnotetext{
Sobre la Declaración de Independencia se han escrito miles de obras; una visión introductiva puede encontrarse por ejemplo en Aparisi Miralles, Ángela, "Los derechos humanos en la Declaración de Independencia de 1776", en Ballesteros, Jesús (editor), Derechos humanos. Concepto, fundamento, sujetos, Madrid, Tecnos, 1992; Asís Roig, Rafael y Ansuátegui Roig, Francisco Javier, "Los derechos humanos en las colonias de Norteamérica” en VV. AA., Historia de los derechos fundamentales, tomo I, Madrid, Dykinson, Universidad Carlos III, 1998 y Asís Roig, Rafael de, Ansuátegui Roig, Francisco J. y Dorado Porras, Javier, "Los textos de las colonias de Norteamérica y las enmiendas a la Constitución" en Historia de los derechos fundamentales, tomo II, volumen III, Madrid, Universidad Carlos III, Editorial Dykinson, 2001.

3 Una aplicación de las divergencias entre Jefferson y Madison sobre el papel de las Constituciones y la posibilidad de reformarlas puede verse en Sunstein, Cass, The Constitution of many minds, Princeton, Princeton University Press, 2009, pp. 1-19.

4 Jefferson, Thomas, Autobiografía y otros escritos, Madrid, Tecnos, 1987, pp. 517-521. Una buena selección del pensamiento del autor puede encontrarse en Jefferson, Thomas, Writings, Nueva York, The Library of America, 1984 (hay reimpresiones posteriores).
} 
haría demasiado mutable como para conservar en su favor los prejuicios que la antigüedad inspira y que tal vez constituyen una saludable ayuda para el más racional de los Gobiernos en la más ilustrada era? ¿ No engendraría tan periódica revisión facciones perniciosas que de otra manera no podrían cobrar experiencia?".

La supervivencia de una forma de gobierno debe hallarse, dice Madison, en la utilidad que le pueda suponer para las generaciones futuras; si dicha utilidad no existe, entonces habrá que cambiar la forma de gobierno, pero eso no es algo que se pueda predeterminar con una temporalidad acotada, como lo propone Jefferson. Sigue Madison: "Las mejoras introducidas por los muertos constituyen una carga para los vivos que sacan de ellas los correspondientes beneficios. Esta carga no se puede satisfacer de otra manera más que ejecutando la voluntad de los muertos que acompañaba a las mejoras".

La conclusión de Madison es que una generación puede y debe obligar a las siguientes, siempre que de tales obligaciones se obtengan beneficios. Sus palabras son las siguientes: "Parece haber fundamento en la naturaleza de las cosas en orden a la relación en que está una generación con otra, en particular en cuanto al traspaso de obligaciones de una a otra. Lo exige la equidad, y de ello derivan beneficios para una y otra generación. Todo lo que es necesario en lo que hace al ajuste de las cuentas entre los muertos y los vivos es ver que las deudas deparadas a éstos no excedan de las ventajas creadas por los primeros"

En las mismas coordenadas temporales, pero del otro lado del Océano Atlántico, se fraguaba en Francia un movimiento constitucional igualmente importante, pero con distintos objetivos. La Revolución francesa no pudo alumbrar, en un primer momento, un texto constitucional, pero aportó el que ha sido calificado como el documento jurídico más importante del mundo moderno ${ }^{6}$ : la Declaración de los Derechos del Hombre y del Ciudadano, del 26 de agosto de 1789. La Declaración fue redactada para servir de preámbulo a lo que luego sería un texto constitucional que ya no pudo ser creado por el mismo cuerpo deliberativo, sino que llegó algunos años después 7 .

Las ideas que animaron el surgimiento de la Declaración no fueron en modo alguno parecidas que aquellas que incendiaron el imaginario de los "founding fathers" en los Estados Unidos. En Francia no se tenía que producir la independencia de ninguna potencia colonial, sino establecer un sistema en el que no reinaran más los privilegios de nacimiento y en el que no hubiera una monarquía absolutista. Se trataba de pro-

Todas las citas provienen de Madison, James, República y libertad, Madrid, CEPC, 2005, pp. 102-103. El mismo documento y otros del mayor interés pueden verse en Madison, James, Writings, Nueva York, The Library of America, 1999.

6 Wachsmann, Patrick, "Déclaration des droits de l'homme et du citoyen" en Alland, Denis y Rials, Stéphane (directores), Dictionnaire de la culture juridique, París, PUF, 2003, pp. 350-351. Miguel Artola califica a la Declaración francesa de 1789 como "la más conocida e influyente de todas las Declaraciones", Los derechos del hombre, Madrid, Alianza, 1986, p. 10.

Un análisis del origen histórico y del significado de la Declaración en el desarrollo de los derechos fundamentales puede verse en Carbonell, Miguel, Una historia de los derechos fundamentales, México, Porrúa, UNAM, CNDH, 2005, páginas 65 y siguientes. 
clamar solemnemente, a través de un documento jurídico, la ruptura de la Revolución con el Antiguo Régimen.

Y vaya que se logró (al menos por unos años, hasta que llegó la restauración monárquica y el prolongado dominio de Napoleón y sus sucesores). Quizá la norma que mejor refleja el ideario rupturista de la Revolución sea el artículo 1 de la Declaración, que señalaba: "Los hombres nacen y permanecen libres e iguales en derechos...."

Otro episodio, otros objetivos y otras formas de constitucionalismo se presentaron muchos años después en la ciudad de Querétaro (México). Se trataba de dotar de estructura jurídica al régimen todavía militar que había surgido de la primera revolución del siglo XX. La aportación del constitucionalismo mexicano quedó plasmada en los artículos que reconocían derechos para campesinos y trabajadores, que establecían un sistema de educación a cargo del Estado para los habitantes del país, que preveían un sistema de propiedad comunal de la tierra.

La discusión que tuvo lugar en el Teatro de la República de Querétaro, organizada a partir del texto presentado el 1 de diciembre de 1916 por el Presidente Venustiano Carranza ${ }^{9}$, terminó alumbrando una Constitución de las clases sociales oprimidas. Comenzaba su andadura el constitucionalismo de signo social. Los constituyentes mexicanos quisieron (aunque luego la realidad los derrotaría clamorosamente) terminar con la exclusión social, política y económica de obreros y campesinos, que habían sido explotados por décadas.

De ese modo llegamos, en esta narración telegráfica, a las Constituciones de la Segunda Posguerra, es decir las Constituciones que han sido identificadas con el neoconstitucionalismo $^{10}$. Son Constituciones, como la italiana de 1947 o la alemana de 1949, que buscan decir "nunca más" a las experiencias de los regímenes genocidas, de signo nazista o fascista. Nunca más a la persecución de minorías religiosas o étnicas, nunca más a la entronización de una "dictadura constitucional", nunca más al genocidio y al atropello de la más elemental dignidad humana.

También en el caso de las Constituciones de Portugal en 1976 y España en 1978 se intentaba dejar atrás las recientes experiencias autoritarias de ambos países. Pero ya

Sobre los alcances de este artículo, Carbonell, Miguel, Una historia de los derechos fundamentales, cit., pp. 106109 .

9 El texto puede verse en Tena Ramírez, Felipe, Leyes fundamentales de México 1808-1989, México, Porrúa, 1989, páginas 764 y siguientes; el texto original aprobado por el constituyente de 1916-1917 se encuentra en Carbonell, Miguel, Cruz Barney, Óscar y Pérez Portilla, Karla, Constituciones históricas de México, 2a edición, México, Porrúa, UNAM, 2004, páginas 493 y siguientes.

10 Una explicación sobre los significados y alcances del concepto pueden verse en Comanducci, Paolo, "'Formas de (neo)constitucionalismo: un análisis metateórico" en Carbonell, Miguel (editor), Neoconstitucionalismo(s), $4^{\text {a }}$ edición, Madrid, Trotta, 2009, páginas 75 y siguientes; también en Carbonell, Miguel, "Neoconstitucionalismo: elementos para una definición”, Jus constitucional. Análisis multidisciplinario de la jurisprudencia del Tribunal Constitucional, Lima, Grijley, número 5, mayo de 2008, pp. 361-372, así como en la presentación de la obra colectiva Teoría del neoconstitucionalismo. Ensayos escogidos, Madrid, Trotta, 2007, pp. 9-12. Una completa exposición del tema y sus implicaciones en García Figueroa, Alfonso, Criaturas de la moralidad. Una aproximación neoconstitucionalista al derecho a través de los derechos, Madrid, Trotta, 2009. 
no para evitar la repetición de un genocidio, sino más bien para abrirse camino hacia la democracia. Construir un régimen con elecciones periódicas, sufragio universal y bien contado, libertades públicas, descentralización.

Se trata de procesos deliberativos parecidos en parte a lo que habría de vivir América Latina en la década de los 80 del siglo pasado ${ }^{11}$. Guatemala en 1985, Brasil en 1988, Colombia en 1991, Argentina a través no de una convención constituyente ni de una nueva Constitución pero sí a través de una reforma integral en 1994. Fueron procesos constituyentes animados y guiados por un puñado de ideas que todavía, tantos años después, siguen teniendo la vigencia de los propósitos no alcanzados: democracia, derechos para todos, equilibrio de poderes, democracia de mercado, combate a la exclusión social y a las desigualdades.

Un capítulo aparte lo representa la Constitución de Sudáfrica de 1996, por su texto y por su contexto, ambos ejemplares y edificantes para las discusiones en torno al nuevo constitucionalismo ${ }^{12}$. La forma de dejar atrás ese régimen esperpéntico del apartheid, de transitar por la ruta casi inédita del perdón sin olvido, la apuesta por la reconciliación racial y política fueron (y siguen siendo) una muestra del enorme poder emancipador y constructor de solidaridades que contiene el constitucionalismo. La Corte Constitucional sudafricana se ha convertido en un referente internacional en materia de protección de derechos sociales, por ejemplo ${ }^{13}$.

La más reciente etapa en esta historia de la odisea constitucional se produce en los años 90 del siglo pasado en Europa del Este (una vez que cae el Muro de Berlín y se derrumba para siempre la lógica bipolar que había regido por décadas las relaciones entre las grandes potencias del Este y el Oeste) y en América Latina.

Las etapas más recientes se encuentran en Venezuela, Ecuador y Bolivia. Los experimentos constitucionales de esos tres países parecen tener como vectores ideológicos tres cuestiones al menos: a) democracia con mayor inclusión social; b) amplios catálogos de derechos fundamentales; y c) fortalecimiento del poder ejecutivo, incluyendo la delicada cuestión (al menos para América Latina, según lo demuestra la historia) de la reelección presidencial ${ }^{14}$.

11 Véanse al respecto las consideraciones de Carpizo, Jorge, Concepto de democracia y sistema de gobierno en América Latina, Bogotá, UNAM, Universidad del Externado de Colombia, 2009; del mismo autor, "Tendencias actuales del constitucionalismo latinoamericano" en Carbonell, Miguel, Carpizo, Jorge y Zovatto, Daniel (coordinadores), Tendencias del constitucionalismo en Iberoamérica, México, IIJ-UNAM, IDEA, AECI, IIDC, 2009, pp. $1-31$.

12 Chaskalson, Matthew y otros, Constitutional law of South Africa, Kenwyn, Juta, 2000.

13 Christiansen, Eric C., "Decidindo sobre direitos nao-justiciaveis: direitos socio-economicos e a Corte Constitucional Sul-Africana” en Pereira de Souza Neto, Claudio y Sarmento, Daniel (coordinadores), Direitos sociais. Fundamentos, judiciliazacao e direitos sociais en especie, Rio de Janeiro, Lumen Iuris, 2010, pp. 647 y siguientes.

14 Véanse las aportaciones que analizan los procesos de renovación constitucional en Venezuela, Ecuador y Bolivia contenidos en la obra Tendencias del constitucionalismo en Iberoamérica, cit.; sobre el caso de Ecuador, Ávila Santamaría, Ramiro (editor), La Constitución de 2008 en el contexto andino. Análisis de la doctrina y el derecho comparado, Quito, Ministerio de Justicia y Derechos Humanos, 2008; Ávila Santamaría, Ramiro, Grijalva Jiménez, Agustín y Martínez Dalmau, Rubén (editores), Desafíos constitucionales. La Constitución ecuatoriana del 2008 en perspectiva, Quito, Ministerio de Justicia y 
Es en este momento en el que se encuentra el constitucionalismo del siglo XXI. Tenemos constituciones muy amplias, respaldadas por consensos sociales robustos y maduros; constituciones que diseńan el ser presente de los países pero sobre todo su deber-ser futuro. Son las utopías concretas de nuestro tiempo.

$\mathrm{Y}$ en este marco es que tiene sentido advertir de los siguientes cuatro desafíos para el constitucionalismo del siglo XXI.

\section{Cuatro desafíos.}

\section{A) Conocer la Constitución.}

Un primer desafío importante para el nuevo constitucionalismo tiene que ver con los procesos cognitivos que se generan y reproducen alrededor o sobre el texto constitucional. Me refiero, para decirlo en pocas palabras, a la posibilidad real de que las constituciones sean conocidas más allá del pequeño círculo de personas que se dedican (profesionalmente) a su estudio o de los aplicadores que deben manejarlas cotidianamente. Que sean conocidas, leídas, apropiadas, por sus destinatarios primeros y últimos, que son todos los habitantes de un país. Puede parecer algo obvio o que deba darse por descontado, pero no considero que sea ni lo uno ni lo otro. Según las encuestas que tenemos disponibles en materia de cultura constitucional, un porcentaje muy alto de personas no conocen nada de la Constitución que rige sus vidas y sus relaciones con los poderes públicos ${ }^{15}$.

No debemos sorprendernos de ello, tomando en cuenta la muy débil cultura política y jurídica que caracteriza desde hace décadas a nuestros ciudadanos, más preocupados por sobrevivir en una realidad que les es adversa que en ocuparse de cartas constitucionales que les suenan muy lejanas y que, en todo caso, les resultan en buena medida ajenas.

Pero además, hay que considerar la dificultad que supone conocer con cierta solvencia textos que, como los latinoamericanos recientes, son bastante largos y prolijos. Pensemos por ejemplo en el caso de una de las Constituciones más recientes, como es la de Ecuador. Dicha Constitución se compone de 444 artículos, 30 disposiciones transitorias y otras 30 del "régimen de transición".

Derechos Humanos, 2008. Un detenido estudio de la Constitución venezolana de 1999 puede verse en Brewer Carías, Allan R., La Constitución de 1999. Derecho constitucional venezolano, 4a edición, Caracas, Editorial Jurídica Venezolana, 2004, 2 tomos.

15 Ver por ejemplo, respecto al caso de México, la obra colectiva Cultura de la Constitución en México. Una encuesta nacional de actitudes, percepciones y valores, México, UNAM, 2004. Hay ejercicios parecidos para los casos de Costa Rica y Argentina; los textos respectivos pueden encontrarse en www. bibliojuridica.org 
En el caso de otros países la dificultad no es tanto por la extensión del texto constitucional, sino por su extrema movilidad a través del mecanismo de enmienda o reforma. México creo que es el caso extremo, aunque no el único. De 1917 a 2009 la Constitución ha sufrido más de 600 modificaciones, las cuales se han plasmado en 189 decretos de reforma (hasta el 16 de noviembre de 2009). De ellos, los últimos 31 decretos han sido publicados en los últimos 3 años, es decir, la velocidad de la reforma ha sido de casi un decreto por mes. Esto, como es obvio, obstaculiza severamente que se pueda conocer, discutir y difundir el contenido de la Constitución, incluso en el caso de los profesionales del derecho, que tienen que comprar ediciones del texto que se publiquen en hojas desechables o sustituibles; es decir, ediciones que permitan dar cuenta de la "motorización" del proceso de reforma constitucional.

Por si lo anterior fuera poco, hay que añadir que el conocimiento de la Constitución es solamente una parte dentro del material que debe conocer cualquier persona interesada en el tema de los derechos fundamentales, por ejemplo. Al texto constitucional hay que añadir esa fuente de primera importancia que son hoy en día los tratados internacionales en materia de derechos humanos, e incluso a las fuentes derivadas de tales tratados, como la jurisprudencia internacional o los documentos emanados de los comités internacionales creados por dichos tratados (por ejemplo, las recomendaciones generales de los comités de Naciones Unidas en temas como los derechos de los niños, la discriminación contra la mujer, los derechos sociales, económicos y culturales, etcétera).

La visión del ordenamiento jurídico como una pirámide en cuyo vértice está la Constitución probablemente ya no sirva para describir los ordenamientos jurídicos modernos, caracterizados por un exuberante pluralismo en el tema de las fuentes del derecho ${ }^{16}$. Quizá sea mejor comenzar a representar al ordenamiento jurídico como un árbol y no como una pirámide.

En todo caso, lo importante es resaltar la evidente necesidad de llevar la Constitución más allá del círculo de los especialistas y de los órganos encargados en lo inmediato y de forma directa de su aplicación. La Constitución del siglo XXI debe romper ese círculo y proyectarse sobre todos los habitantes del respectivo Estado. De esa forma se podrá en verdad hablar de patriotismo constitucional más allá de la retórica.

16 Al tema del "pluralismo de las fuentes del derecho" en el Estado constitucional de nuestros días le ha dedicado varias páginas brillantes Gustavo Zagrebelsky; por ejemplo en sus obras, Manuale di diritto costituzionale. Il sistema delle fonti del diritto, Turín, UTET, 1993 (reimpresión), El derecho dúctil. Ley, derechos, justicia, $3^{a}$ edición, Madrid, Trotta, 1999 y La legge e la sua giustizia, Bolonia, Il Mulino, 2008. Me he referido al mismo tema en Carbonell, Miguel, Constitución, reforma constitucional y fuentes del derecho en México, 6a edición, México, Porrúa, UNAM, 2008 capítulo I. 


\section{B) Aplicarla bien.}

La presencia tan relevante de principios y de cláusulas abiertas en los nuevos textos constitucionales, a la que hemos hecho referencia en el apartado anterior, conlleva el reto de aplicar bien tales disposiciones, lo que supone al menos las siguientes dos cuestiones:

a) Dotar de sentido concreto, aplicable a casos concretos, a esas cláusulas abiertas, semánticamente vagas e indeterminadas ${ }^{17}$.

Tomemos como ejemplo, nuevamente, el caso de la Constitución de Ecuador, que hoy en día es el mejor ejemplo (el más logrado técnicamente, me parece) de las Constituciones del neoconstitucionalismo en América Latina. Conforme avanzamos en su lectura nos encontramos con ese tipo de cláusulas indeterminadas y abiertas: hay referencias a un Estado de derechos y justicia (artículo 1), a un medio ambiente sano y equilibrado (artículo 14), a una comunicación incluyente y participativa (artículo 16), a una información oportuna, contextualizada y plural (artículo 18), a una vivienda digna y adecuada (artículo 30), a la atención integral a la salud (artículo32), etcétera.

Lo mismo sucede con otros muchos textos constitucionales de la región. Por ejemplo, quien lea la Constitución brasileña de 1988 se encontrará con conceptos tan indeterminados como los de dignidad de la persona (artículo 1.III), pluralismo político (artículo 1.V), igualdad ante la ley (artículo 5), proporcionalidad en el derecho de respuesta (artículo 5.V), intimidad, vida privada y honor (artículo 5.X), derechos adquiridos (artículo 5.XXXVI), pena cruel (artículo 5.XLVII inciso E), debido proceso legal (artículo 5.LIV), etcétera.

Obviamente, se podría hacer un ejercicio semejante respecto de casi todas las Constituciones recientes y el resultado sería muy parecido.

Pues bien, el intérprete de esas normas debe ser capaz de dotarlas de un contenido concreto, de "aterrizarlas", a partir seguramente de una adecuada teoría de la norma jurídica (dada la necesidad de trabajar con principios, como modelo normativo distinto al de las reglas, con las que se trabaja en otras ramas del conocimiento jurídico) ${ }^{18}$.

b) la aplicación compleja tendiente a dotar de sentido a las normas abiertas, a la que acabamos de hacer referencia, presupone y exige construir una buena teoría de la argumentación. Una teoría que permita a los aplicadores de las normas jurídicas ser capaces de operar en la práctica con conceptos y técnicas interpretativas tan sofisticados como la proporcionalidad, la ponderación, el efecto irradiación, la eficacia horizontal

17 Sobre la ambigüedad y la vaguedad de las normas jurídicas en general, así como sobre las dificultades que comportan para la interpretación, Guastini, Riccardo, Estudios sobre la interpretación jurídica, $6^{a}$ edición, México, Porrúa, UNAM, 2004, pp. 57 y siguientes.

18 La distinción entre principios y reglas, que es un tema central de las discusiones constitucionales contemporáneas, puede verse por ejemplo en Atienza, Manuel y Ruiz Manero, Juan, Las piezas del derecho, $2^{a}$ edición, Barcelona, Ariel, 2004, capítulo I. Una discusión sobre el tema, pero aplicada al campo de la interpretación, en Guastini, Riccardo, Nuovi studi sull'interpretazione, Roma, Aracne, 2008, pp. 119 y siguientes. 
de los derechos fundamentales, el principio pro-personae, la aplicación prevalente de los tratados internacionales (sobre estos dos últimos puntos puede verse el interesante artículo 417 de la Constitución de Ecuador), el principio de coherencia, el principio de universalidad de los derechos, etcétera.

Todo lo anterior se debe hacer teniendo presente la idea general de la aplicación directa de las normas constitucionales, es decir, la no necesidad de que exista siempre y en todos los casos intermediación legislativa para poder aplicar una norma constitucional a un caso concreto (una idea que forma parte de lo que Riccardo Guastini ha llamado las "condiciones de la constitucionalización del ordenamiento jurídico") ${ }^{19}$.

Otra idea general a tomar en cuenta en este contexto es el de la "interpretación conforme", de acuerdo con la cual el intérprete (sobre todo en sede jurisdiccional), puede salvar la constitucionalidad de una norma legislativa, pero proporcionando a la vez la orientación para que sea correctamente interpretada, de conformidad con lo que señala la Constitución ${ }^{20}$.

Igualmente, es muy posible que los intérpretes constitucionales, al construir sus argumentaciones, deban tener una cierta apertura hacia el conocimiento científico ${ }^{21}$. Algunos tribunales constitucionales del mundo están incorporando cada vez con mayor frecuencia las evidencias científicas a sus sentencias, de modo que se reconoce el lugar que la ciencia puede y debe tener en el proceso, incluyendo por supuesto al proceso constitucional.

Tanto la concretización constitucional como la argumentación en la materia, dan lugar (o deben dar lugar) a jueces activistas. El activismo judicial no significa ni implica que el juez pueda sustituir por su criterio personal las decisiones que ha tomado el constituyente $^{22}$. Eso solamente sucede en las versiones caricaturizadas que hacen del activismo sus enemigos. El activismo judicial, bien entendido, significa simplemente que el juez toma todas las normas constitucionales en serio y las lleva hasta el límite máximo que permite su significado semántico, a fin de proteger con la mayor extensión normativa y fáctica los derechos fundamentales. Precisamente, el activismo será más marcado en materia de derechos y mucho más moderado (o deferente) cuando se trate de cuestiones relativas a la división de poderes o al ejercicio de competencias públicas.

El activismo judicial no deviene o surge de una postura académica o ideológica, sino que -me parece- es parte del modelo mismo de la democracia constitucional, tal

19 Guastini, Riccardo, "La constitucionalización del ordenamiento jurídico: el caso italiano" en Carbonell, Miguel (editor), Neoconstitucionalismo(s), 4a edición, Madrid, Trotta, 2009, p. 55-56.

20 Guastini, Riccardo, "La constitucionalización del ordenamiento jurídico: el caso italiano", cit., pp. 56-57.

${ }_{21}$ Ver sobre el tema, de entre lo mucho que se ha escrito, el ensayo de Michele Taruffo, "Conocimiento científico y estándares de prueba judicial”, Jueces para la democracia, número 52, Madrid, marzo de 2005, pp. 63-73.

22 Una interesante exposición sobre el tema, realizada además por una persona con varias décadas de experiencia práctica, puede verse en Barak, Aharon, The judge in a democracy, New Haven, Yale University Press, 2006; también es importante revisar lo que se afirma sobre el tema en Ferreres, Víctor, Justicia constitucional y democracia, Madrid, CEPC, 1997, así como en la obra colectiva Jueces y derecho. Problemas contemporáneos, 2a edición, México, Porrúa, UNAM, 2008. 
como es entendido al menos desde el surgimiento del neoconstitucionalismo (al día siguiente del final de la Segunda Guerra Mundial). La democracia constitucional debe contar con jueces vigilantes, custodios intransitables e intransigentes de los derechos fundamentales; jueces que estén dispuestos y bien preparados para llevar las normas que prevén tales derechos hasta sus últimas consecuencias, maximizando su contenido normativo ${ }^{23}$.

Si revisamos los casos más conocidos de activismo judicial, podemos identificar claramente las enormes aportaciones que han hecho los jueces que lo personifican (y podemos también darnos cuenta que los riesgos sobre los que nos advierten sus críticos existen en su imaginación más que en la realidad).

Si recurrimos a los ejemplos históricos más conocidos veremos que las etapas de fuerte activismo judicial se han dado sobre todo en relación a los derechos fundamentales. Los jueces activistas han concentrado su actuación en generar las condiciones para reconocer una dimensión fuertemente normativa (a veces incluso de carácter prestacional, dependiendo del caso) a los derechos fundamentales.

Tomemos el ejemplo más conocido sobre un Tribunal activista: la Suprema Corte de los Estados Unidos mientras fue presidida por Earl Warren, entre 1953 y $1969^{24}$. ¿Qué fue lo que hicieron ese grupo de jueces que no fuera estrictamente apegado al paradigma irrenunciable del Estado constitucional? Si revisamos sus más conocidas sentencias veremos que, lejos de asumir funciones que no les correspondían, los justices de la Corte Warren se limitaron a aplicar, pero con todas sus consecuencias, lo que con claridad se podía deducir del texto constitucional vigente.

En ese entonces se generaron importantes precedentes en materia de igualdad racial en las escuelas (caso Brown versus Board of Education de 1954)25, de la supremacía

23 Desde luego, el activismo que es propio del modelo del Estado constitucional no es el "activismo cuantitativo" que Gustavo Zagrebelsky ha denunciado en una de sus elocuentes reflexiones sobre el papel de los tribunales constitucionales; el activismo cuantitativo es la "mera productividad" del tribunal, en cuya consecución se ponen todas las energías de sus miembros, como si su tarea fuese la de sacar el mayor número de asuntos para poder presentar, a final de ańo, una estadística satisfactoria para quienes no entienden para qué puede servir la jurisdicción constitucional. Zagrebelsky apunta que hay que resistir siempre esta tentación por los números gruesos. Tiene razón, ya que las grandes épocas de la jurisdicción constitucional no son recordadas precisamente por el número de sentencias dictadas, sino por su impacto en la sociedad. La Suprema Corte de los Estados Unidos, que como quiera es un ejemplo del que mucho se puede y se debe aprender, no emite más de 70 u 80 sentencias al ańo. Pero el impacto de cada una de ellas no tiene parangón en casi ningún otro país del mundo. Ver sobre el "activismo cuantitativo" el apunte de Zagrebelsky, Gustavo, Principios y votos. El Tribunal Constitucional y la politica, Madrid, Trotta, 2008, p. 43.

24 Sobre la Corte Warren y sus más destacadas sentencias, así como sobre sus integrantes, existen centenares de libros y quizá miles de artículos publicados. Ha sido una de las etapas de la Suprema Corte de los Estados Unidos que ha merecido una mayor atención de los especialistas. Para una primera aproximación puede ser de utilidad revisar el libro de Tushnet, Mark (editor), The Warren court in historical and political perspective, Charlottesville, Londres, Virginia University Press, 1993. Una muy completa biografía del propio Earl Warren puede verse en Newton, Jim, Justice for all. Earl Warren and the nation he made, Nueva York, Riverhead Books, 2006.

25 Patterson, James, Brown versus Board of education. A civil rights milestone and its troubled legacy, Nueva York, Oxford University Press, 2001; Kluger, Richard, Simple justice. The history of Brown v. Board of Education and black America's struggle for equality, Nueva York, Vintage Books, 2004; Cottrol, Robert J. y otros, Brown v. Board of Education. Caste, culture and the Constitution, Lawrence, Kansas University Press, 2003. 
judicial en la interpretación de la Constitución (Cooper versus Aaron de 1958), de cateos y revisiones policíacas (caso Mapp versus Ohio de 1961) ${ }^{26}$, de libertad religiosa (caso Engel versus Vitale de 1962) ${ }^{27}$, de asistencia letrada gratuita (caso Gideon versus Wainwrigth de 1963) ${ }^{28}$, de libertad de prensa (New York Times versus Sullivan de 1964) ${ }^{29}$, de derechos de los detenidos (Miranda versus Arizona de 1966) o de derecho a la intimidad de las mujeres (Griswold versus Connecticut de 1965 en relación con la compra y el uso de métodos anticonceptivos) ${ }^{30}$.

¿Quién se atrevería a sostener que la igualdad racial en las escuelas, la posibilidad de realizar críticas vehementes a los funcionarios públicos o el derecho a la asistencia letrada gratuita en materia penal no forman parte del corazón mismo del modelo del Estado Constitucional de derecho? ¿Dónde está, por tanto, el exceso de la que se reconoce como la Corte más activista del mundo? No hay tal exceso ni mucho menos una situación de riesgo para los valores y derechos tutelados constitucionalmente, salvo en la imaginación de aquellos que objetan las tareas judiciales como una forma (poco) encubierta de denostar al modelo mismo, que es en realidad lo que les molesta, dadas las muchas exigencias y controles que de él derivan.

El activismo judicial ha permitido alcanzar lo que algunos teóricos han denominado la "revolución de los derechos" 31 , pero lo interesante (para la teoría general del derecho constitucional y, sobre todo, para la teoría constitucional latinoamericana), es que tal revolución ha sido acompańada por elementos adicionales sobre los que conviene reparar.

En otras palabras, el activismo judicial ha sido una condición o elemento esencial para la revolución de los derechos si atendemos a la evidencia histórica disponible, pero no el único. Hay que contar además con buenas declaraciones de derechos, con marcos institucionales que permitan "activar" la actuación de los jueces (acciones populares,

University Press, 2006.

27 Dierenfield, Bruce J., The battle over school prayer. How Engel v. Vitale changed America, Lawrence, Kansas University Press, 2007.

28 Lewis, Anthony, Gideon's trumpet, Nueva York, Vintage Books, 1989.

29 Lewis, Anthony, Ninguna ley. El caso Sullivan y la primera enmienda, Miami, SIP, 2000.

30 Johnson, John W., Griswold v. Connecticut. Birth control and the constitutional right of privacy, Lawrence, Kansas University Press, 2005; Tribe, Lawrence, Abortion. The clash of absolutes, Nueva York, Londres, Norton and Company, 1992. Con independencia de su valor e interés intrínseco, la sentencia Griswold es recordada sobre todo porque fue el precedente invocado por la Corte al resolver el caso más importante en materia de interrupción voluntaria del embarazo: Roe versus Wade de 1973. En Griswold la Corte dedicó buena parte de su sentencia a justificar la existencia de un "derecho a la intimidad" derivado de otros derechos establecidos explícitamente en algunas de las enmiendas que conforman el Bill of rights. Para la Corte los derechos establecidos por el Bill of rights tienen zonas de "penumbra", de las cuales emanan otros derechos que ayudan a los primeros a tener vida y sustancia. En Griswold se estaba discutiendo la constitucionalidad de una ley del Estado de Connecticut, del año de 1879 , que impedía la difusión de información y el uso de anticonceptivos; la Corte afirmó que esa ley violaba la intimidad de las parejas y se preguntó: “¿Permitiremos a la policía vulnerar los sagrados precintos de las recámaras maritales para encontrar evidencias del uso de contraceptivos? Esta simple idea es repulsiva para la noción de privacidad que rodea a la relación matrimonial".

31 Epp, Charles R., The rights revolution. Lawyers, activists and Supreme Courts in comparative perspective, Chicago y Londres, The University of Chicago Press, 1998. 
legitimación activa amplia, amicus curiae, etcétera), con una fuerte conciencia social alrededor de los derechos, etcétera.

Me interesa detenerme en el último de los aspectos mencionados: la conciencia social alrededor de los derechos y la correspondiente existencia de un fuerte "activismo social" para su defensa. En efecto, al margen de los alcances que pueda tener una judicatura decidida a llevar hasta sus últimas consecuencias la interpretación constitucional, lo cierto es que los jueces no deberían ser los únicos y ni siquiera los más destacados intérpretes de las constituciones. La interpretación constitucional debe correr a cargo, como lo ha señalado Peter Häberle, de una "sociedad abierta de los intérpretes constitucionales" 32 . En este sentido, podemos constatar que las experiencias de fuerte activismo judicial suelen estar acompañadas de ciertas dosis de "activismo social", mayor o menor dependiendo del contexto de que se trate, pero casi siempre presente; ese activismo social cancelaría las críticas que sostienen que la actuación garantista (o activista) de los jueces contiene riesgos anti-democráticos ${ }^{33}$.

Para que tal activismo social se pueda dar es necesario no solamente que la sociedad o algunos de sus grupos se mantengan alertas en la defensa de sus derechos, sino también que el ordenamiento jurídico permita traducir en pretensiones procesales esa vigilancia. Esto suena como algo muy obvio para lectores de países avanzados, pero supone un problema de considerables dimensiones en países que sufren de graves retrasos en la evolución de sus ordenamientos jurídicos, en los que no suele existir vía alguna para que desde la sociedad se emprendan acciones judiciales contra políticos corruptos o contra administraciones públicas que amparadas en la opacidad violan cotidianamente los derechos fundamentales de un buen número de ciudadanos.

La historia constitucional de los Estados Unidos permite vislumbrar, aparte de un modelo de constitucionalismo que presenta muchas peculiaridades y que ha sido paradigmático para un número importante de países, las condiciones que son necesarias para realizar una revolución jurídica a partir de los derechos fundamentales, contando con fuertes dosis de activismo judicial ${ }^{34}$.

En realidad, en Estados Unidos se han producido dos revoluciones de los derechos. La primera, a finales del siglo XVIII cuando en las constituciones de las colonias se comenzaron a "constitucionalizar" los derechos fundamentales, cumpliendo con la primera etapa del desarrollo histórico de los derechos. Ese primer movimiento se completa con el establecimiento del Bill of rights a nivel federal en 1791 y con las enmiendas constitucionales expedidas al finalizar la Guerra Civil a mediados del siglo XIX. Esa es la primera revolución de los derechos que se realiza en territorio norteamericano y, junto con la experiencia francesa de 1789 , la primera en sentido moderno a nivel mundial.

Häberle, Peter, El Estado constitucional, México, UNAM, 2001, pp.149 y siguientes.

Epp, Charles R., The rights revolution. Lawyers, activists and Supreme Courts in comparative perspective, cit., p. 5.

34 Epp, Charles R., The rights revolution. Lawyers, activists and Supreme Courts in comparative perspective, cit., pp. 26 y siguientes. 
La segunda revolución se inicia en la década de los 50, a través de los grandes pronunciamientos de la "Corte Warren" (a los cuales ya hemos hecho referencia). El disparo de salida, preparado durante años por importantes movimientos cívicos en defensa de los derechos, puede fijarse convencionalmente en el año de 1954, cuando se emite la sentencia del caso Brown versus Board of Education para remover la segregación racial en las escuelas. Su realización completa se lleva a cabo desde ese año y hasta la primera mitad de la década de los 70, cuando las fuerzas conservadoras sustituyen en el estrado de la Suprema Corte a los jueces liberales que habían tenido un papel destacado en defensa de los derechos.

La decisión final de esta segunda revolución tal vez haya sido la sentencia del caso Roe versus Wade sobre el derecho de la mujer a interrumpir voluntariamente su embarazo, dictada en 1973. Desde luego, con posterioridad a esa sentencia ha habido otros casos de activismo de gran importancia, pero han sido más escasos, más repartidos en el tiempo y sin que el conjunto de todos ellos haya reproducido en extensión y en intensidad los múltiples pronunciamientos progresistas que en materia de derechos fundamentales fueron dictados entre 1954 y 1973, sobre todo mientras presidía la Corte Earl Warren.

Otros casos de cortes o tribunales activistas pueden encontrarse, por citar solamente dos ejemplos adicionales, en España, con la primera integración del Tribunal Constitucional y hasta el retiro de la mayor parte de los grandes juristas que lo conformaron (entre 1981 y 1992), y en Colombia, con la Corte Constitucional que se crea a partir de la nueva Constitución de 1991 (la Corte dicta sentencias verdaderamente emblemáticas por su progresismo, sobre todo entre 1992 y 1996, cuando se da una formidable actuación de varios magistrados, entre los que cabe destacar a Carlos Gaviria) ${ }^{35}$.

\section{C) Educar para el (neo)constitucionalismo.}

Un enorme desafío para el nuevo constitucionalismo latinoamericano tiene que ver con la formación de cuadros profesionales que sean capaces de operar con solvencia en las coordenadas que han sido descritas en los dos apartados anteriores (me refiero a las consideraciones que se han hecho sobre la "concretización" del texto constitucional y sobre la construcción de pautas argumentativas apropiadas e idóneas para el Estado constitucional de nuestros días).

En esta tarea formativa tienen una gran responsabilidad los centros universitarios de nuestros países, desde los cuales no podemos dejar de preguntarnos: ¿¿ómo enseñamos derecho constitucional?, ¿qué tipo de abogados constitucionalistas necesitamos formar?, ¿cómo destacar la importancia del litigio de interés público, tan relevante en materia de derechos fundamentales, ante los alumnos?, ¿qué metodología debemos emplear

35 Algunos de sus pronunciamientos más importantes se encuentran en Gaviria Díaz, Carlos, Sentencias. Herejías constitucionales. Bogotá, FCE, 2002. 
en las tareas propias de la enseñanza de los derechos fundamentales o de las nuevas formas de división del poder? ${ }^{36}$

Para estar en aptitud de hacer frente a este tercer desafío debemos hacernos cargo de la necesidad de generar una doctrina constitucional propia, que tome lo mejor que se ha producido en otras latitudes, pero que a la vez sea capaz de responder a nuestros propios dilemas y necesidades. Tenemos que seguir leyendo y reflexionando sobre lo que han escrito Ronald Dworkin, Luigi Ferrajoli, Robert Alexy, Gustavo Zagrebelsky, Riccardo Guastini, Luis Prieto, Mark Tushnet, Cass Sunstein, Bruce Ackerman, Laurence Tribe, Jeremy Waldron, José Juan Moreso, Juan Carlos Bayón, Manuel Atienza, etcétera. Pero también debemos contribuir por medio de análisis propios, pertinentes a una realidad que no se parece demasiado a la que han tenido presente tales teóricos, dadas las evidentes distancias que separan a nuestros países de los suyos.

Si revisamos, de nueva cuenta, la experiencia de sistemas constitucionales que podemos calificar como exitosos o al menos como consolidados y robustos, nos daremos cuenta que en casi todos los casos se ha generado una doctrina constitucional propia muy potente. Es el caso del impresionantemente rico discurso constitucional estadounidense (hay docenas de libros publicados en los Estados Unidos sobre todos y cada uno de los aspectos importantes del constitucionalismo contemporáneo) o de la muy solvente doctrina que se genera desde los años 80 del siglo pasado en España, a partir de las publicaciones periódicas y no periódicas del Centro de Estudios Constitucionales de la Presidencia del Gobierno de Espańa (hoy llamado Centro de Estudios Políticos y Constitucionales) $)^{37}$.

\section{D) Contar con la sociedad.}

Podemos tener una buena difusión social de los contenidos constitucionales, podemos haber realizado los mejores ejercicios de concretización constitucional (contando para ello con muy buenas pautas argumentativas), podemos tener jueces activistas y una buena formación de los profesionales que se dedican a aplicar los textos constitucionales con seriedad, rigor y vocación de servicio al interés general, pero nada de ello será útil o podrá generar el efecto deseado si no somos capaces a la vez de cambiar

36 He intentado analizar algunas de estas cuestiones en Carbonell, Miguel, La enseñanza del derecho, $3^{\mathrm{a}}$ edición, México, Porrúa, UNAM, 2008.

37 Lo llamativo del desarrollo de la teoría constitucional en Espańa es que se produjo con una gran velocidad, de forma casi intempestiva. Recordemos que con anterioridad a los ańos 80 , cuando el Tribunal Constitucional de ese país inicia su andadura y cuando se da la etapa más activista que ha tenido, solamente destacaban las aportaciones de Manuel García Pelayo (sobre todo su libro de Derecho constitucional comparado). Los grandes autores del constitucionalismo español apenas comenzaban a rendir sus primeros frutos (Rubio Llorente, Manuel Aragón, Pedro Cruz Villalón) y estaban lejos de ver la luz los textos que a la postre han marcado un antes y un después en la teoría constitucional espańola; el único que ya se había publicado para entonces era el conocido ensayo de Eduardo García de Enterría sobre la Constitución como norma y el tribunal constitucional (aparecido en parte en el número 1 de la Revista Española de Derecho Constitucional, ya desde entonces dirigida por Rubio Llorente). 
la "percepción social" alrededor del derecho y de los abogados. Dicha percepción, al menos en América Latina, no es para nada positiva, lo cual es una de las razones que explica la bajísima adhesión que suscitan las normas jurídicas.

También en el ámbito constitucional debemos hacernos cargo de la muy extendida anomia que caracteriza a nuestras sociedades ${ }^{38}$. Predominan las conductas directa o indirectamente contrarias a las normas jurídicas. La trampa, la evasión, la informalidad, el aprovechamiento de los huecos del sistema, el fraude a la ley y otros ejemplos que se podrían citar se multiplican por millones cotidianamente ${ }^{39}$.

Quienes nos dedicamos profesionalmente al derecho debemos ser capaces de contestar a la pregunta: ¿por qué los ciudadanos deben obedecer las normas jurídicas? Y no lo podemos hacer desde las coordenadas teóricas tradicionales, al menos no si nuestro marco de reflexión es América Latina, donde toda la experiencia histórica de siglos rema en contra de las más básicas justificaciones del deber de obediencia al derecho que se suelen citar en Europa, por ejemplo.

En nuestro caso creo que hay que comenzar explicando y difundiendo el enorme potencial emancipador, casi revolucionario, que tiene el derecho constitucional. Tomada en serio y aplicada con rigor, la normatividad constitucional que ya está vigente en nuestros países (aunque permanezca inactuada en la mayor parte de los casos), puede producir cambios de la mayor profundidad en el corto plazo. La legalidad constitucional debe ser vista hoy en día como el poder de los sin poder, como la palanca más potente para el cambio social, incluso -en muchos contextos- como la única palanca a nuestro alcance.

Como lo ha señalado Paolo Flores D’Arcais, la legalidad aplicada de forma coherente y sin fisuras es, en nuestro tiempo, la primera de las revoluciones posibles, y quizá la única de las revoluciones deseables; sus palabras son las siguientes. "Hoy la legalidad tomada en serio, la legalidad como estrategia y práctica coherente, constituye más que nunca el poder de los sin poder... una política de legalidad es hoy la más radical de las revoluciones posibles, además de la primera de las revoluciones deseables... porque es preliminar a cualquier otra" ${ }^{40}$. No nos demoremos en comenzar a dirigirnos, sin temor y con toda esperanza, hacia la ruta que nos permita llevar a cabo esa revolución pacífica.

38 Una extensa narración de este fenómeno, dedicada a la Argentina, pero fácilmente exportable al resto de países de América Latina, en Nino, Carlos, Santiago, Un país al margen de la ley, Buenos Aires, Ariel, 2005 (reimpresión).

39 Ver el excelente cuadro (excelente por completo, aunque deprimente por sus contenidos y por la evidencia empírica aportada) que nos ofrece García Villegas, Mauricio, Normas de papel. La cultura del incumplimiento de reglas, Bogotá, Siglo del Hombre editores, 2009.

40 Flores D’Arcais, Paolo, El individuo libertario. Recorridos de filosofía moral y politica en el horizonte de lo finito, Barcelona, Seix Barral, 2001, p. 146. 
